\title{
Clinical effectiveness of the pocket-creation method for colorectal endoscopic submucosal dissection $\square$
}

\section{다(1) $(\odot$}

\author{
Authors \\ Hideyuki Hiraishi ${ }^{1}$ \\ Institutions \\ 1 Department of Gastroenterology, Dokkyo Medical \\ University, Tochigi, Japan \\ 2 Department of Diagnostic Pathology, Dokkyo Medical \\ University, Tochigi, Japan
}

Akira Kanamori ${ }^{1}$, Masakazu Nakano${ }^{1}$, Masayuki Kondoํㅜ, Takanao Tanaka ${ }^{1}$, Keiichiro Abe ${ }^{1}$, Tsunehiro Suzuki ${ }^{1}$, Hitoshi

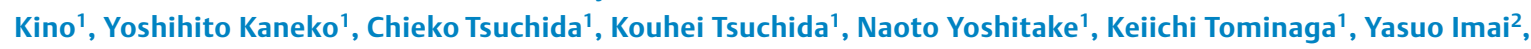

submitted 10.5 .2017

accepterd after revision 14.7 .2017

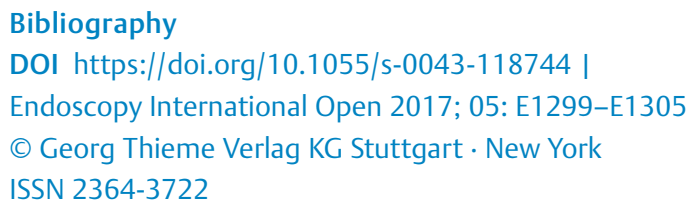

Corresponding author

Masakazu Nakano, Department of Gastroenterology, Dokkyo Medical University, 880, Kitakobayashi, Mibu, Tochigi 321-0293, Japan

Fax: +81282867761

nakano-m@dokkyomed.ac.jp

\section{ABSTRACT}

Background and study aims Endoscopic submucosal dissection (ESD) is a technically advanced procedure for colorectal tumors. Hayashi et al. invented the "pocket-creation method (PCM)," and reported that Is-type lesions with fi- brosis could be efficaciously and safely resected. However, only case studies have been published, and there are no previous reports on the usefulness of PCM in colorectal ESD for all lesions, as compared with the conventional method. This study aimed to evaluate the effectiveness and safety of PCM in colorectal ESD.

Patients and methods Ninety-six colorectal tumors were treated: 47 using the PCM and the other 49 , considered the control group, using the conventional method. Therapeutic effectiveness and safety were retrospectively assessed.

Results The comparison between the PCM and control groups revealed higher rates of en bloc resection (100\% vs. $88 \%, P=0.015)$ and curative endoscopic resection (100\% vs. $84 \%, P=0.0030$ ) with PCM. There was no significant difference in perforation as an adverse event (AE) between the two groups, though perforation was observed in only $6 \%$ of the control group and none of the PCM group. Compared with the control group, the PCM group had lower incidences of perforation and post-ESD coagulation syndrome, and both AEs were associated with excessive thermal denaturation of the muscle layer ( $2 \%$ vs. $16 \%, P=0.018$ ).

Conclusions This study demonstrated the effectiveness and safety of ESD with PCM for colorectal tumors. Although there is a possible learning curve, PCM enables the endoscopist to safely perform ESD in most cases without encountering the difficulties associated with conventional ESD.

\section{Introduction}

Early lesions of colorectal tumors can be cured by endoscopic mucosal resection (EMR), but en bloc resection is difficult for large ( $\geq 20 \mathrm{~mm}$ ) superficial tumors. Local recurrence is thought to be associated with imperfect piecemeal resection, and endoscopic submucosal dissection (ESD) is required to determine the degree of radical cure and to obtain a detailed pathological diagnosis $[1,2]$. ESD is technically more difficult to perform for colorectal tumors than for esophageal and gastric tumors, and the incidence of adverse events (AEs) is known to be higher: a previous report showed the incidences of perforation and delayed bleeding to be $4.9 \%$ and $1.5 \%$, respectively [3]. To perform ESD safely and efficiently, it is important to maintain good visualization of the operative field by pulling the lesion upward during dissection. The usefulness of traction methods such as the clip-flap method, clip and snare method with the prelooping technique, thread-traction method, and S-O clip method has been reported [4-7]. Hayashi et al. invented the "pocket-creation method" (PCM) in which the submucosal layer is dissected to create a wide pocket under a lesion after a minimal mucosal incision. They reported a case study in which a 
giant subpedunculated neoplastic lesion with colorectal fibrosis and laterally spreading tumor (LST) could be resected effectively and safely $[8,9]$. Moreover, according to a report by Miura et al. on the efficacy of PCM in ESD for duodenal tumors, the perforation rate was significantly lower with PCM than with the conventional method, and Sakamoto et al. reported the usefulness of PCM for the nongranular type of colorectal LST $[10,11]$. However, the effectiveness and safety of PCM versus the conventional method for all lesions treated with colorectal ESD have not been previously reported. Thus, the aim of this study was to evaluate the effectiveness and safety of PCM in colorectal ESD.]

\section{Patients and methods}

We investigated 96 patients with colorectal tumors, comprising a total of 96 lesions. For cases with multiple ESDs, we selected the first ESD for analysis. Forty-seven consecutive lesions treated with colorectal ESD through PCM in the Division of Gastroenterology of Dokkyo Medical University between February 2016 and October 2016 comprised the PCM group. PCM was introduced in the Division of Gastroenterology of Dokkyo Medical University in February 2016, and used for all lesions from March 2016 on as a routine treatment strategy for colorectal ESD. Fifty-two consecutive lesions were treated by using conventional ESD between December 2014 and February 2016. After excluding 3 lesions treated by trainees, 49 lesions served as the control group for comparison with the PCM group. The included lesions were colorectal LST or Is-type tumors $\geq 20 \mathrm{~mm}$ in diameter. Absence of tumor invasion into the deep submucosal layer was preoperatively confirmed using chromoendoscopy and image-enhanced endoscopy and, if appropriate, magnification endoscopy. ESD for the 100 lesions was conducted by 2 experienced gastroenterologists who had performed more than 50 colorectal ESD procedures as of December 2014.

As the endpoints of this study, we retrospectively compared the PCM group and the control group in terms of en bloc resection, curative endoscopic resection, resection size, procedure time, dissection speed, and the amount of sodium hyaluronate solution injected as measures of usefulness. Occurrence of perforation, post-ESD coagulation syndrome (CS), and delayed bleeding were considered AEs. In this study, en bloc resection was defined as that achieved by ESD without using a snare, and curative endoscopic resection was defined as en bloc resection with negative resection margins [12]. Procedure time was defined as the time from the start of the injection under the mucosa in order to raise the mucosa around the lesion upward until tumor extraction. Dissection speed was calculated using the following formula: resection size $\left(\mathrm{mm}^{2}\right)$ /procedure time (min). Perforation was defined as a full-thickness defect of the muscular layer, i. e., a state in which connective tissues, the abdominal cavity, or serosa were recognizable [12]. Post-ESD CS refers to post-ESD abdominal pain syndrome caused by thermocoagulation of the intestinal mucosa [13-16]. The diagnostic standards for post-ESD CS were as follows: 1) localized abdominal tenderness and 2) fever $\geq 37.6^{\circ} \mathrm{C}$ or an increased inflammatory response (WBC 10,000 cells/ $\mu \mathrm{L}$ or $C R P \geq 0.5$ ). Delayed bleeding was defined as overt bleeding requiring endoscopic therapy or blood transfusion [12]. Histological features, such as the affected area and gross diameter, were determined in accordance with the academic guidelines [17]. We reviewed medical records, operative reports, and endoscopic and pathological findings. This study was approved by the bioethics committee of our institution and written informed consent was obtained from all patients.

We used PCF Q260 J (OLYMPUS, Tokyo, Japan) or GIF Q260 J (OLYMPUS) as the endoscope, and ST Hood (DH-15GR; Fujifilm, Tokyo, Japan) and Distal Attachments (D-201; OLYMPUS) as the endoscopic hoods for the PCM group and the control group, respectively. Dual knife (OLYMPUS) was routinely used, and, if appropriate, the Hook knife (OLYMPUS) and IT knife nano (OLYMPUS) combination was used to incise the mucosa or dissect the submucosal layer. Coagrasper (OLYMPUS) was used as hemostatic forceps. The solution to be injected was prepared by adding a small amount of indigo carmine dye and adrenaline to a $0.4 \%$ sodium hyaluronate solution (Mucoup; Boston Scientific, Japan). VIO300D (ERBE Elektromedizin Ltd, Tübingen, Germany) was used as the high-frequency electrical generator. Midazolam and pentazocine hydrochloride were administered intravenously for sedation during surgery. Butylscopolamine bromide or glucagon was used as an anticonvulsant. Carbon dioxide $\left(\mathrm{CO}_{2}\right)$ was used as an insufflation gas. After resection of the lesions, minimum coagulation/hemostasis was performed using hemostatic forceps for the exposed blood vessels at the bottom of the tumors in order to prevent procedural bleeding.

- Fig. 1 and P Fig. 2 show the ESD procedure using PCM $(\triangleright$ Video 1). Alternatively, the conventional ESD procedure was performed in the control group, in retroflex view when possible. After local injection into the submucosal layer, a mucosal incision was created at the oral side of the lesion. Dissection was conducted, after additional and sufficient local injection into the submucosal layer, to avoid perforation. Local injection and dissection were repeated to complete the submucosal layer dissection.

\section{Statistical analysis}

Age and tumor size as a patient background factor and tumor characteristic were compared with the Mann-Whitney $U$ test. Gender, tumor location, growth type, and histology were compared using the chi-squared test. In the analysis of differences in treatment results, the Fisher's exact test was used for en bloc resection, curative endoscopic resection, and AEs; the Mann-Whitney $U$ test was used to compare resection size, procedure time, dissection speed, and the amount of injected sodium hyaluronate solution under the mucosa. En bloc resection, curative endoscopic resection, and perforation were estimated with $95 \%$ confidence intervals ( $95 \% \mathrm{Cls}$ ), based on the exact binominal distribution. $P$ values $<0.05$ were considered indicative of a statistically significant difference, and StatFlex version 6.0 (Artech Co., Osaka, Japan) was used for statistical analysis. 


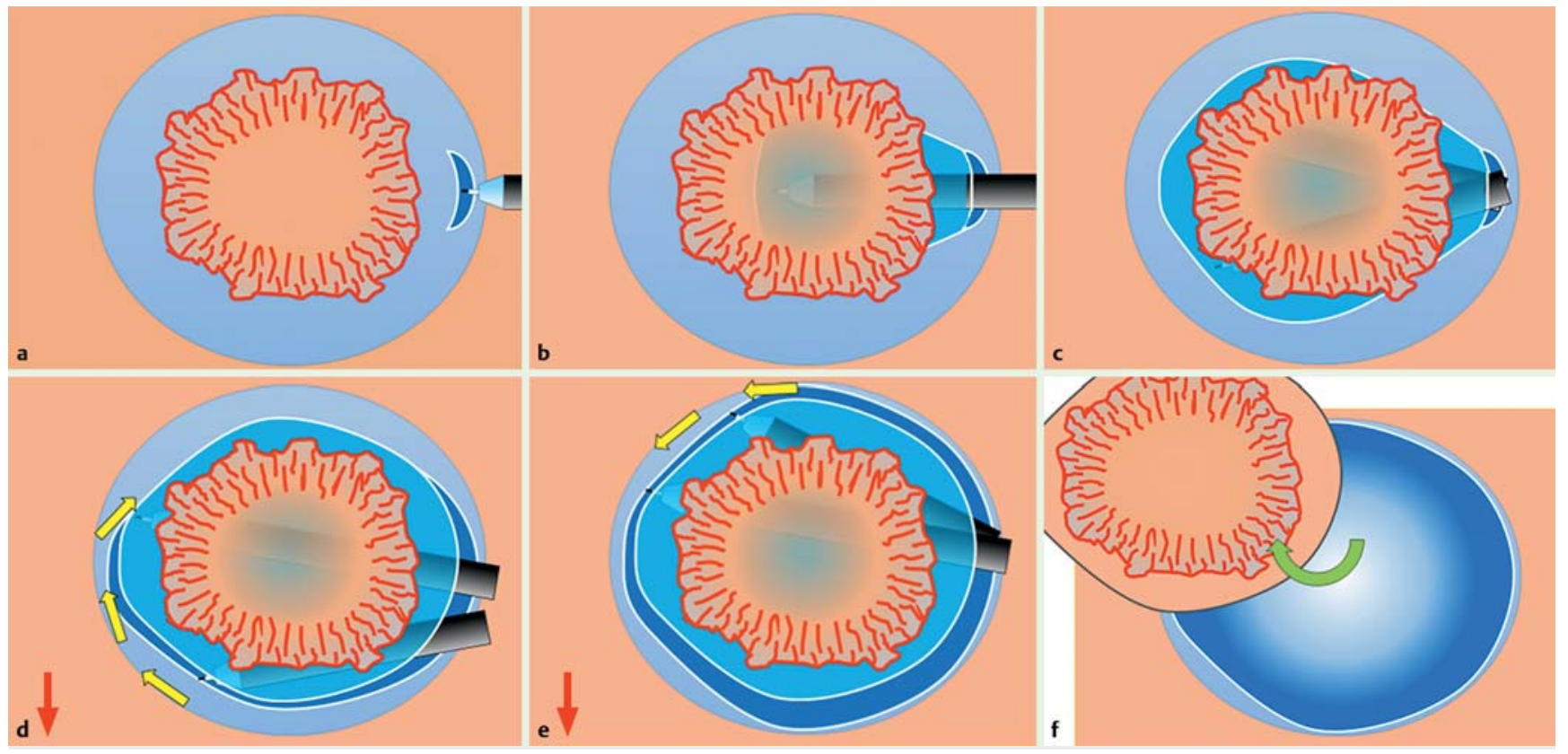

- Fig. 1 Schematic of pocket creation [7-9]. Red arrows in some images show the direction of gravity. a A minimal mucosal incision is made following a submucosal injection. b, c A large submucosal pocket under the tumor. $\mathbf{d}$ The pocket is opened in a step-by-step manner working toward the distal side and against gravity. e The remaining area is dissected. $\mathbf{f}$ An en bloc resection is accomplished.

\section{Results}

- Table 1 shows patient backgrounds and tumor characteristics. There were no significant differences in age, gender, tumor location, tumor size, growth type, histology, or fibrosis between the two groups. - Table 2 shows the clinical outcomes. The PCM group had significantly better outcomes for en bloc resection ( $100 \%$ vs. $88 \%, P=0.015 ; 95 \% \mathrm{Cl} 92-100 \%, 75-95 \%$ ) and curative endoscopic resection ( $100 \%$ vs. $84 \%, P=0.0030$; $95 \% \mathrm{Cl} 92-100 \%, 70-93 \%)$ than did the control group. No significant difference was observed in procedure time $(77 \mathrm{~min}$ vs. $85 \mathrm{~min}, P=0.38)$ or dissection speed $\left(14.3 \mathrm{~mm}^{2} / \mathrm{min}\right.$ vs. $\left.11.8 \mathrm{~mm}^{2} / \mathrm{min}, P=0.57\right)$. All 6 lesions that were not resected en bloc occurred in the control group. There was no significant difference in perforation as an $\mathrm{AE}$, although perforation occurred only in the control group with no perforation cases observed in the PCM group (6\% vs. $0 \%, P=0.13 ; 95 \% \mathrm{Cl} 1-17 \%, 0-8 \%$ ). Three patients who experienced intraprocedural perforation showed improvement with additional conservative treatment after the perforation had been successfully closed with an endoscopic clip. No significant difference was observed in the frequency of post-ESD CS between the 2 groups ( $2 \%$ vs. $10 \%$, $P=0.11)$. All 6 patients with post-ESD CS improved with additional conservative treatment. No significant difference in the incidence of perforation and post-ESD CS as AEs was observed between the PCM and control groups. However, as compared with the control group, the PCM group had lower incidences of perforation and post-ESD CS, which are AEs associated with excessive thermal denaturation of the muscle layer ( $2 \%$ vs. $16 \%$, $P=0.018)$. No difference was found in the incidence of delayed bleeding between the two groups ( $9 \%$ vs. $8 \%, P=0.62$ ). The amount of injected sodium hyaluronate solution under the mu- cosa was smaller in the PCM group than in the control group (30 mL vs. $38 \mathrm{~mL}, P=0.020$ ).

\section{Discussion}

This study successfully demonstrated the effectiveness and safety of ESD with PCM. All patients undergoing ESD with PCM remained free of perforation and achieved en bloc and curative resection. The $\mathrm{PCM}$ procedure can be completed with an antegrade approach only and does not require scope inversion.

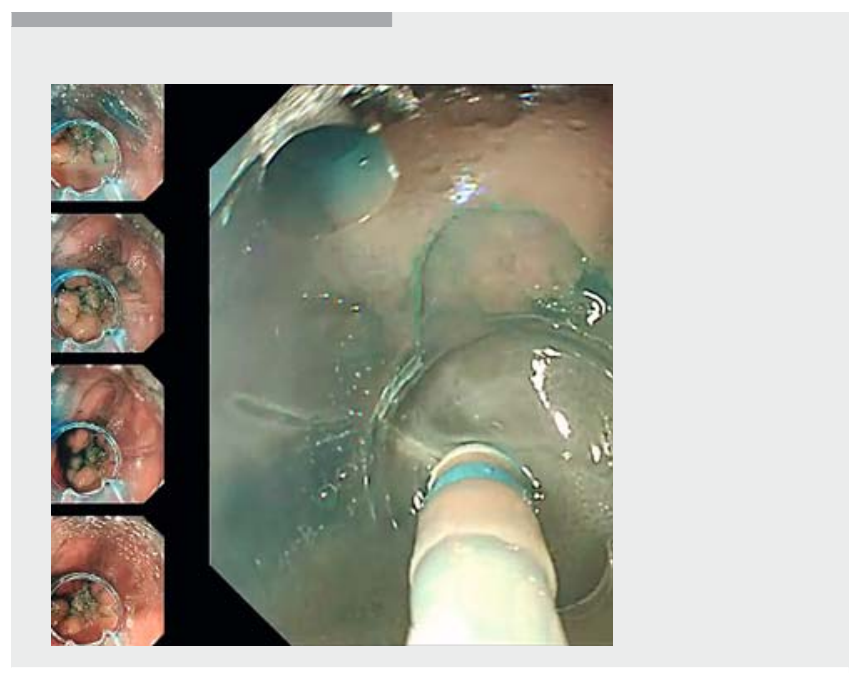

$\checkmark$ Video 1 A colorectal endoscopic submucosal dissection procedure using the pocket-creation method for laterally spreading tumor - granular type. 

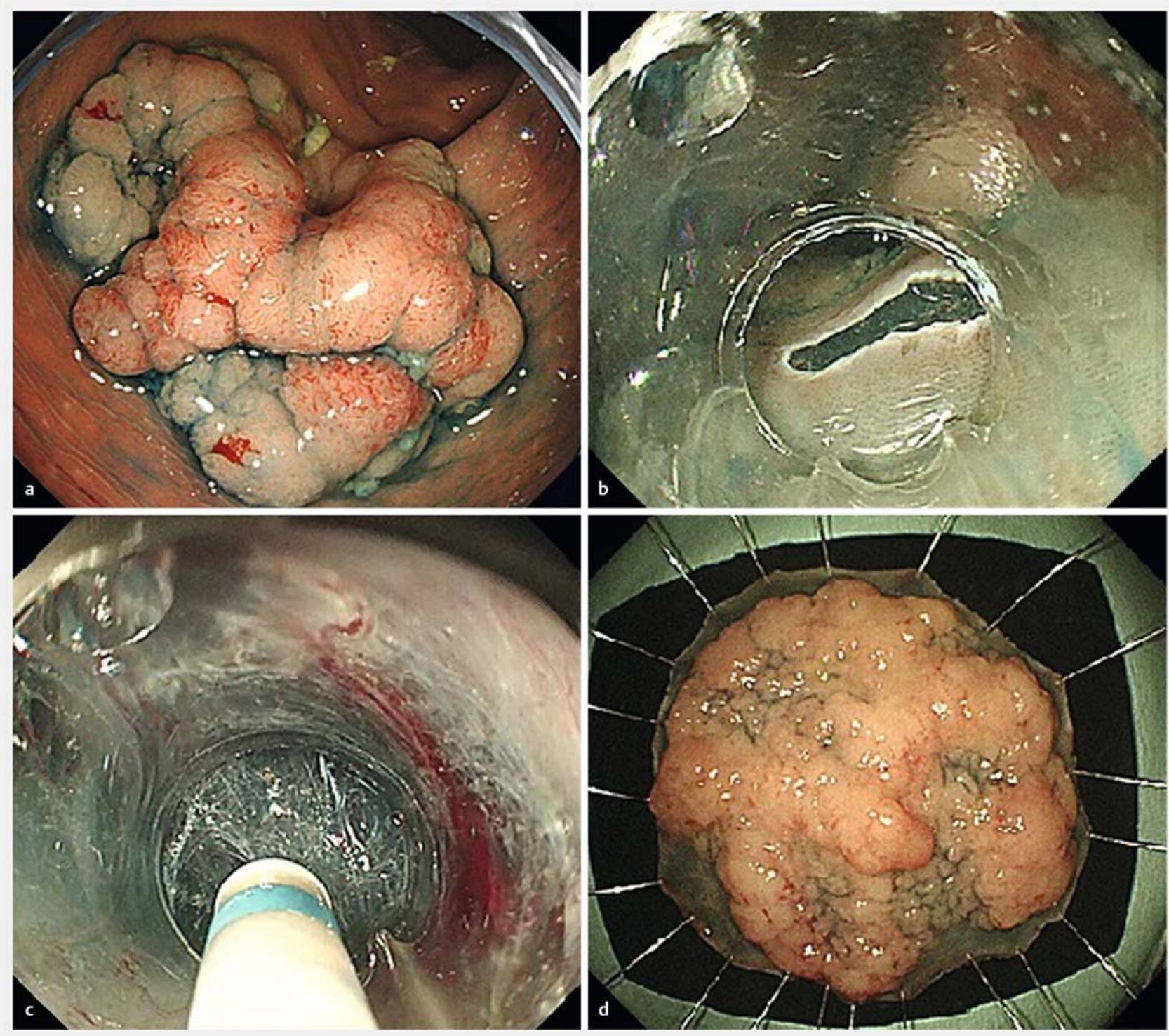

- Fig. 2 ESD using PCM for colorectal tumors is shown. a Laterally spreading tumor (granular type) $48 \times 45 \mathrm{~mm}$ in size is recognizable in the sigmoid colon. b A minimal mucosal incision was made following a submucosal injection. c An ST hood, the tip of the endoscope, was inserted into the layer to be dissected and dissection of the submucosal layer was conducted with a dual knife. $\mathbf{d}$ A resected specimen $51 \times 51 \mathrm{~mm}$ in size was obtained and diagnosed as tubular adenoma. The vertical and horizontal margins were negative with no lymphovascular invasion.

Hori et al. defined difficult ESD cases as those requiring a long procedure time, piecemeal resection, and resulting in perforation, and reported that: 1 ) tumors with scarring or local recurrence; 2) tumors $\geq 50 \mathrm{~mm}$ in size or spreading across $\geq 2$ folds; and 3) flexure location was an independent risk factor in difficult ESD cases [18]. They also pointed out in previous reports that fibrosis was the most significant risk factor for perforation caused by colorectal ESD $[19,20]$. The effectiveness of traction methods such as the clip-flap method, clip and snare method with the prelooping technique, thread-traction method, and S-O clip method has been reported to enable a safer and more effective performance of high-level colorectal ESD [4-7]. However, these methods are not cost-efficient and entail the problem of endoscope reinsertion. Furthermore, the preparations are generally more complicated, since adjuvant devices are required. In contrast, PCM does not require additional devices, allowing for the simple and efficient traction of all lesions. In PCM, as the endoscopic hood extends to the lower and upper layers of the dissected submucosa, in addition to gravity, not only traction but also countertraction could also be achieved [8-11]. Thus, PCM can be used routinely as a standard treatment strategy for colorectal ESD regardless of the difficulty of the procedure. Of the 6 cases that did not achieve en bloc resection in this investigation, all were in the control group. Endoscopic treatment was discontinued in 2 patients who were unable to continue due to intraprocedural perforation, and in another 2 in whom dissection was not possible due to fibrosis of the submucosal layer. The latter 2 patients 
were switched to piecemeal resection using a snare after discontinuation of ESD because it was difficult to maintain the tangential approach to the muscle layer due to cecal involvement and strong respiratory movements. Hayashi et al. reported the following advantages of PCM: 1) stable visualization can be maintained; 2) the efficiency of dissection increases by stretching the tissues; and 3) the tangential approach to the muscle layer can be used even at a fold or a flexure $[8,9]$. According to our actual results, PCM provided stable visualization, increased the efficiency of dissection by stretching tissues via traction, and facilitated the tangential approach to the muscle layer, enabling the safe completion of ESD without perforation even in 18 lesions involving lateral tumor spread (nongranular type) with fold convergence, or various fibroses such as post-biopsy scarring and post-EMR scarring. Even for lesions in patients with strong respiratory movements that reduce endoscope maneuverability or with vertical cecal involvement, continuation of the submucosal layer dissection was possible since stable visualization was maintained by inserting the endoscope into the submucosal layer.

As compared to the control group, the PCM group had lower incidences of perforation and post-ESD CS, AEs associated with excessive thermal denaturation of the muscle layer. Post-EMR CS was reported in a study by Waye et al., and excessive thermocoagulation of the muscle layer by high-frequency electrosurgical energy during EMR has been shown to cause serosal inflammation [21]. Coagulation syndrome caused by excessive coagulation was also reportedly observed in $9.5 \%$ to $40.2 \%$ of cases undergoing colorectal ESD [13-16]. This might be because PCM facilitates the tangential approach to the muscle layer such that there is less possibility of causing thermal denaturation of the muscle layer due to a high-frequency wave, and also lower incidences of perforation and CS associated with
- Table 1 Characteristics of the patients and tumors.

\begin{tabular}{|c|c|c|c|}
\hline & $\begin{array}{l}\text { PCM group } \\
(n=47)\end{array}$ & $\begin{array}{l}\text { Control } \\
\text { group } \\
(n=49)\end{array}$ & $P$ value \\
\hline Age, median (range) & $70(41-83)$ & $71(44-83)$ & 0,32 \\
\hline Gender, male (\%) & $32(68)$ & $33(67)$ & 0,94 \\
\hline Tumor location, n (\%) & & & 0,68 \\
\hline - Right side of colon & $25(53)$ & $30(61)$ & \\
\hline - Left side of colon & $12(26)$ & $8(16)$ & \\
\hline - Rectum & $10(21)$ & $11(22)$ & \\
\hline $\begin{array}{l}\text { Tumor size, median } \\
\text { (range), mm }\end{array}$ & $26(20-68)$ & $30(20-58)$ & 0,21 \\
\hline - Growth type, n (\%) & & & 0,42 \\
\hline . LST-G & $32(68)$ & $32(65)$ & \\
\hline . LST-NG & $13(28)$ & $12(24)$ & \\
\hline - Others (\%) & $2(4)$ & $5(10)$ & \\
\hline Histology, n (\%) & & & 0,24 \\
\hline - Adenoma & $40(85)$ & $37(76)$ & \\
\hline - Carcinoma & $7(15)$ & $12(24)$ & \\
\hline Fibrosis, n (\%) & $18(38)$ & $18(37)$ & 0,87 \\
\hline
\end{tabular}

LST-G, laterally spreading tumor - granular type; LST-NG, laterally spreading tumor - non-granular type.

\section{- Table 2 Clinical outcomes.}

\begin{tabular}{|c|c|c|c|}
\hline & $\begin{array}{l}\text { PCM group } \\
(n=47)\end{array}$ & $\begin{array}{l}\text { Control group } \\
(n=49)\end{array}$ & $P$ value \\
\hline En-bloc resection, n (\%) & $47(100)$ & $43(88)$ & 0,015 \\
\hline Curative endoscopic resection, n (\%) & $47(100)$ & $41(84)$ & 0,0030 \\
\hline Resection size, mm & $32(22-75)$ & $35(21-67)$ & 0,27 \\
\hline Procedure time, minutes & $77(10-256)$ & $85(28-335)$ & 0,38 \\
\hline Dissection speed, $\mathrm{mm}^{2} / \mathrm{min}$ & $14.3(3.6-54.2)$ & $11.8(4.9-36.9)$ & 0,57 \\
\hline Perforation, n (\%) & $0(0)$ & $3(6)$ & 0,13 \\
\hline - Intraprocedural, n (\%) & $0(0)$ & $3(6)$ & \\
\hline - Delayed, n (\%) & $0(0)$ & $0(0)$ & \\
\hline Post-ESD CS, n (\%) & $1(2)$ & $5(10)$ & 0,11 \\
\hline Perforation or Post-ESD CS, n (\%) & $1(2)$ & $8(16)$ & 0,018 \\
\hline Delayed bleeding, n (\%) & $4(9)$ & $4(8)$ & 0,62 \\
\hline Sodium hyaluronate solution, $\mathrm{mL}$ & $30(7-114)$ & $38(12-92)$ & 0,020 \\
\hline
\end{tabular}


thermal denaturation of the muscle layer than when using the conventional method.

PCM is considered advantageous in that there is only a small amount of unnecessary leakage of the injected solution due to the minimal incision and a good bulge can be obtained under the mucosa $[8,9]$. We used sodium hyaluronate in this study for the local submucosal injection in colorectal ESD, and found that the injected amount was actually significantly smaller in the PCM group. Use of the PCM avoids unnecessary repeated local injections and thereby a reduction in ESD time can be expected. Considering that a bottle of sodium hyaluronate $(20 \mathrm{~mL})$ is relatively expensive $(7,740$ yen) in Japan, PCM is anticipated to reduce healthcare costs. Although procedure times and the speed of specimen dissection were not improved in this study, these treatment results have been obtained in the early phase of PCM introduction, and further improvement can be expected with accumulated experience.

One disadvantage of PCM is the slight difficulty in maintaining visualization during hemostasis if blood pools within a pocket when bleeding occurs during ESD. In this investigation, hemostasis for intraoperative bleeding was achieved in all cases, but prior confirmation of the blood vessels present in the layer to be dissected under the mucosa within a pocket, as well as sufficient pre-coagulation for large blood vessels, were necessary. Another disadvantage of PCM is that the dissection area of the submucosal layer must be unnecessarily enlarged for particularly small lesions; consequently, the dissected specimens were sometimes unnecessarily large, since incision of the adjacent mucosa is the last step. Endoscopists should be careful not to excessively widen the dissection area of the submucosal layer within the pocket.

Our study has 3 limitations. First, it study was conducted in only 1 institution. Multicenter studies are necessary to generalize the usefulness of PCM. Second, this was a retrospective study. Randomized controlled trials are required to confirm the efficacy of PCM. Finally, both endoscopists were highly experienced, which reduced bias but affected the learning curve. Therefore, many endoscopists with different experience levels, including trainees, should be involved in future investigations. As the 2 groups of this study had different observation periods, there is a possibility of a learning curve.

\section{Conclusion}

In conclusion, this investigation showed that PCM is effective for all lesions because it enables the safe completion of colorectal ESD in most cases without the difficulties encountered in conventional ESD.

\section{Acknowledgements}

We thank the staff of the Division of Gastrointestinal Endoscopy, Dokkyo Medical University Hospital for their contribution to this study.
Competing interests

None

References

[1] Hotta K, Fujii T, Saito Y et al. Local recurrence after endoscopic resection of colorectal tumors. Int J Colorectal Dis 2009; 24: 225-230

[2] Saito Y, Fukuzawa M, Matsuda T et al. Clinical outcome of endoscopic submucosal dissection versus endoscopic mucosal resection of large colorectal tumors as determined by curative resection. Surg Endosc 2010; 24: $343-352$

[3] Saito Y, Uraoka T, Tamaguchi Y et al. A prospective, multicenter study of 1111 colorectal endoscopic submucosal dissections (with video). Gastrointest Endosc 2010; 72: 1217-1225

[4] Yamamoto K, Hayashi S, Saiki H et al. Endoscopic submucosal dissection for large superficial colorectal tumors using the "clip-flap method". Endoscopy 2015; 47: 262-265

[5] Yamada S, Doyama H, Ota R et al. Impact of the clip and snare method using the prelooping technique for colorectal endoscopic submucosal dissection. Endoscopy 2016; 48: $281-285$

[6] Oyama T. Counter traction makes endoscopic submucosal dissection easier. Clin Endosc 2012; 45: 375-378

[7] Ritsuno H, Sakamoto N, Osada T et al. Prospective clinical trial of traction device-assisted endoscopic submucosal dissection of large superficial colorectal tumors using the S-O clip. Surg Endosc 2014; 28: $3143-3149$

[8] Hayashi Y, Sunada K, Takahashi H et al. Pocket-creation method of endoscopic submucosal dissection to achieve en bloc resection of giant colorectal subpedunculated neoplastic lesions. Endoscopy 2014; 46: 421-422

[9] Hayashi Y, Miura Y, Yamamoto H. Pocket-creation method for the safe, reliable, and efficient endoscopic submucosal dissection of colorectal lateral spreading tumors. Dig Endosc 2015; 27: 534-535

[10] Miura Y, Shinozaki S, Hayashi Y et al. Duodenal endoscopic submucosal dissection is feasible using the pocket-creation method. Endoscopy 2016: doi:s-0042-116315 (Epub ahead of print)

[11] Sakamoto H, Hayashi Y, Miura Y et al. Pocket-creation method facilitates endoscopic submucosal dissection of colorectal laterally spreading tumors, non-granular type. Endosc Int Open 2017; 05 : E123-E129

[12] Tanaka S, Kashida H, Saito Y et al. JGES guidelines for colorectal endoscopic submucosal dissection/endoscopic mucosal resection. Dig Endosc 2015; 27: 417 - 434

[13] Yamashina T, Takeuchi Y, Uedo $\mathrm{N}$ et al. Features of electrocoagulation syndrome after endoscopic submucosal dissection for colorectal neoplasm. J Gastroenterol Hepatol 2016; 31: 615-620

[14] Jung D, Youn YH, Jahng J et al. Risk of electrocoagulation syndrome after endoscopic submucosal dissection in the colon and rectum. Endoscopy 2013; 45: 714-717

[15] Hirasawa K, Sato C, Makazu M et al. Coagulation syndrome: Delayed perforation after colorectal endoscopic treatments. World J Gastrointest Endosc 2015; 7: 1055 - 1061

[16] Hong M], Kim JH, Sung IK et al. Prevalence and clinical features of coagulation syndrome after endoscopic submucosal dissection for colorectal neoplasms. Dig Dis Sci 2015; 60: 211-216

[17] Watanabe T, Itabashi M, Shimada $Y$ et al. Japanese Society for Cancer of the Colon and Rectum (JSCCR) guidelines 2010 for the treatment of colorectal cancer. Int J Clin Oncol 2012; 17: 1-29 
[18] Hori K, Uraoka T, Harada K et al. Predictive factor for technically difficult endoscopic submucosal dissection in the colorectum. Endoscopy 2014; 46: $862-870$

[19] Takamaru H, Saito Y, Yamada M et al. Clinical impact of endoscopic clip closure of perforations during endoscopic submucosal dissection for colorectal tumors. Gastrointest Endosc 2016; 84: 494-502
[20] Isomoto H, Nishiyama H, Yamaguchi N et al. Clinicopathological factors associated with clinical outcomes of endoscopic submucosal dissection for colorectal epithelial neoplasms. Endoscopy 2009; 41: $679-683$

[21] Waye JD. The post polypectomy coagulation syndrome. Gastrointest Endosc 1981; 27: 184 